\title{
La calidad peatonal como método para evaluar entornos de movilidad urbana
}

\author{
Ruben Talavera-Garcia ${ }^{1}$ \\ Julio Alberto Soria-Lara ${ }^{2}$ \\ Luis Miguel Valenzuela-Montes ${ }^{1}$ \\ rtalaverag@ugr.es \\ j.a.sorialara@uva.nl \\ lvmontes@ugr.es
}

(1) Laboratorio de Planificación Ambiental (LABPLAM)

Universidad de Granada. Departamento de Urbanística y Ordenación del Territorio

(2) University of Amsterdam. Amsterdam Institute for Social Sciences Research

Universidad de Zaragoza. Departamento de Geografía y Ordenación del Territorio

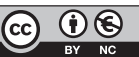

Recepción: septiembre de 2012

Aceptación: noviembre de 2012

\section{Resumen}

El creciente interés por integrar elementos de la estructura y el diseño de la ciudad en la evaluación y la gestión de la movilidad urbana ha contribuido al desarrollo de nuevos métodos e instrumentos que profundicen en este aspecto. La identificación de entornos de movilidad podría considerarse un avance conceptual y metodológico importante en este sentido, puesto que abre nuevas posibilidades para la generación de métodos que comprendan mejor la vinculación entre movilidad y entorno urbano desde una perspectiva que pueda ser útil en la práctica de la planificación.

Este artículo se dirige a profundizar sobre la cuestión apuntada, particularizando en el caso específico de la movilidad peatonal. De este modo, se presenta el método de caracterización peatonal de entornos de movilidad (CPEM) como herramienta útil para evaluarlos desde el punto de vista de su calidad peatonal, utilizando los cuatro factores siguientes: accesibilidad, seguridad, confort y atracción. Para comprobar la consistencia y la utilidad de CPEM respecto a su objetivo inicial, éste ha sido aplicado al caso práctico de dos entornos de movilidad previamente identificados en un corredor del área metropolitana de Granada (España).

Palabras clave: entornos de movilidad; calidad peatonal; diseño urbano.

Resum. La qualitat per als vianants com a mètode per avaluar entorns de mobilitat urbana

El creixent interès per integrar elements de l'estructura i el disseny de la ciutat en l'avaluació i en la gestió de la mobilitat urbana ha contribuït a desenvolupar mètodes i instruments 
nous que aprofundeixin en aquest aspecte. La identificació d'entorns de mobilitat es podria considerar un avenç conceptual i metodològic important en aquest sentit, que obre noves possibilitats per a la generació de mètodes que comprenguin millor la vinculació entre mobilitat i entorn urbà des d'una perspectiva que pugui ser útil per practicar la planificació.

Aquest article es dirigeix a aprofundir sobre la qüestió apuntada, particularitzant en el cas específic de la mobilitat de vianants. D’aquesta manera, es presenta el mètode de caracterització d'entorns de mobilitat per a vianants (CPEM) com a eina útil per avaluarne des del punt de vista de la qualitat que mostren per als vianants, utilitzant els quatre factors següents: accessibilitat, seguretat, confort i atracció. Per comprovar la consistència i la utilitat del CPEM respecte al seu objectiu inicial, aquest ha estat aplicat al cas pràctic de dos entorns de mobilitat prèviament identificats en un corredor de l'àrea metropolitana de Granada (Espanya).

Paraules clau: entorns de mobilitat; qualitat per als vianants; disseny urbà.

Résumé. Qualité des piétons comme une méthode pour l'évaluation des environnements de mobilité urbaine

L'intérêt chaque fois plus grand, pour intégrer les éléments de la structure et le design de la ville en l'évaluation et aménagement de la mobilité urbaine, a contribué au développement de nouveaux méthodes et outils qui approfondissent cet aspect. L'identification des environnements de mobilité pourrait être considéré comme une avancée conceptuelle et méthodologique importante dans ce sens, ce qui ouvre de nouvelles possibilités pour générer des méthodes permettant de mieux comprendre le lien entre la mobilité et de l'environnement urbain dans une perspective qui peut être utile dans la pratique de planification.

Cet article prétend approfondir cette question, en particularisant dans le cas spécifique de la mobilité piétonnière. De cette façon, on présente la méthode de Caractérisation Piétonnière d'Environnements de Mobilité (CPEM) comme une outil pour évaluer les environnements de mobilité dans le point de vue de sa qualité piétonnière, en utilisant les quatre facteurs suivants: accessibilité, sécurité, confort et attraction. Pour vérifier la consistance et l'utilité de CPEM par rapport à son objectif initial, on a appliqué au cas pratique de deux «environnements de mobilité» au préalable identifiés dans un corridor de l'agglomération urbaine de Grenade (Espagne).

Mots clé: environnements de mobilité; qualité piétonnière; dessin urbain.

\section{Abstract. Pedestrian quality as a method to evaluate urban mobility environments}

Over the last years there has been a growing interest to integrate structure, urban design and travel patterns. The incorporation of this knowledge into practice, namely mobility planning, has been positively affected by the development of new planning tools. Despite the innovation of mobility environment approach as a novel instrument for mobility planning, new methods seem to be needed. They aim to deepen our understanding of the relationships between urban structure and travel pattern through mobility environment concept.

The article aims to gain insight into this problem using the particular case of pedestrian mobility. A method to characterize the pedestrian quality of mobility environments is presented here (CPEM). CPEM assesses the pedestrian quality through four walkability aspects: accessibility, security, comfort and attractiveness. To illustrate the worth of this method, a practical implementation to the context of the metropolitan area of Granada, Spain, is presented. The paper describes this case, explores the advantages and disadvantages of CPEM, and finally anticipates some issues for future research.

Keywords: mobility environments; pedestrian quality; urban structure. 


\section{Sumario}

\section{Introducción}

2. Factores determinantes de la calidad peatonal de los entornos de movilidad

3. Método para caracterizar la calidad peatonal de entornos de movilidad (CPEM)
4. Aplicación del método CPEM y resultados obtenidos

5. Discusión y conclusiones

6. Agradecimientos

Referencias bibliográficas

\section{Introducción}

Innovar en la gestión de la movilidad urbana exige enfrentarse a un creciente número de demandas, que oscilan desde la dimensión más socioambiental hasta una vertiente puramente urbanístico-tecnológica. De ahí la necesaria y creciente preocupación por desarrollar métodos y herramientas capaces de integrar tales demandas, tanto a nivel conceptual y metodológico (Banister, 2008), como a nivel instrumental (Vieira et al., 2007). El paradigma predict and prevent frente al clásico predict and provide (Bertolini et al., 2008; Herce, 2009) ha contribuido fuertemente a la valoración desde una perspectiva integrada de las distintas componentes que conforman la movilidad urbana (Givoni y Banister, 2010), esencialmente desde la consideración de la estructura de la ciudad como uno de los factores motrices de la necesidad de viajar y, consecuentemente, del patrón de viaje (Silva y Pinho, 2010).

En este contexto, han sido varios los trabajos que han aparecido en la literatura especializada que han profundizado sobre el uso de unidades espaciales para articular la toma de decisiones en el campo de la movilidad urbana, tanto desde una perspectiva vinculada a la movilidad motorizada (Cervero, 2002; Delmelle y Casas, 2012), como desde una aproximación más cercana a la movilidad peatonal o a través de modos alternativos (Clifton et al., 2007). En la mayoría de casos, estos trabajos crecen apoyados sobre estudios e informes que sostienen la hipótesis de la existencia de un fuerte vínculo entre elementos de la estructura urbana y los patrones de viaje en la ciudad (Crane, 2000; Ewing y Cervero, 2010; Foltête y Piombini, 2007; Handy y Niemeier, 1997). De este modo, evoluciona, a nivel conceptual (Bertolini y Dijst, 2003) y metodológico, (Soria, 2011) la idea de entorno de movilidad como base espacial sobre la que desarrollar un enfoque integrado - al menos entre estructura urbana y patrón de viaje- de la movilidad urbana, situación que ha llevado, en el caso específico de la movilidad no motorizada, a hablar de entornos peatonales como de un modelo basado en aquellos factores urbanos condicionantes de la movilidad peatonal (topografía, arbolado, actividad comercial, seguridad, etc.) y también en el predominio lógico de este modo de desplazamiento (Borst et al., 2009; Zacharias, 2001) en dichos entornos.

A priori, la identificación de entornos de movilidad podría considerarse un paso adelante para una integración efectiva entre la estructura urbana y los 
patrones de viaje, en el marco de la planificación y la gestión de la movilidad (Rodríguez et al., 2009; Soria et al., 2010) y, específicamente, en el contexto de la movilidad no motorizada (Clifton et al., 2007). Sin embargo, parece necesario profundizar sobre el desarrollo de métodos que ayuden a caracterizar y entender mejor el funcionamiento de tales entornos de movilidad, de manera que éstos puedan llegar a convertirse en instrumentos cada vez más precisos en la toma de decisiones. Esta necesidad se debe, esencialmente, a dos cuestiones:

1. Los indicadores y los procedimientos utilizados para identificar entornos de movilidad no siempre son los más idóneos para la interpretación y la comprensión posteriores de su funcionamiento.

2. Adoptar medidas o estrategias concretas para un entorno de movilidad determinado, por ejemplo, respecto del desplazamiento peatonal, exige utilizar indicadores y métodos específicos que aporten mayor rigor en el proceso de planificación.

Este artículo se dirige a profundizar sobre la caracterización y la evaluación de entornos de movilidad desde el punto de vista de su calidad peatonal. Para ello, se presenta el método CPEM (caracterización peatonal de entornos de movilidad). Su intención es la de profundizar sobre la problemática apuntada midiendo la calidad peatonal a partir de los cuatro aspectos siguientes: accesibilidad, seguridad, confort y atracción ciudadana. Con el fin de evaluar su utilidad y consistencia, el modelo CPEM será aplicado al caso práctico de dos entornos de movilidad en un corredor del área metropolitana de Granada (España), donde las instituciones competentes debaten sobre la implantación de un sistema de metro ligero como factor de atracción peatonal entre otros muchos aspectos.

Tras esta introducción (primer apartado), el artículo se estructura en los cuatro bloques siguientes: el segundo apartado recoge los principales fundamentos teóricos sobre los que se asienta la investigación; el tercer apartado presenta el método para caracterizar la calidad peatonal de los entornos de movilidad (CPEM); el cuarto apartado aplica, paso a paso, dicho método a dos entornos de movilidad en el área metropolitana de Granada (España); finalmente, el quinto apartado realiza una discusión de los resultados obtenidos y expone las principales conclusiones del artículo.

\section{Factores determinantes de la calidad peatonal de los entornos de movilidad}

\subsection{Entornos de movilidad}

Las interrelaciones existentes entre la estructura urbana y el patrón de viaje no solo son complejas, sino que cuentan con una gran cantidad de dimensiones e interacciones posibles. Esto ha hecho que un amplio número de autores haya intentado diseccionar la relación o las relaciones existentes entre ambos 
conceptos respecto a la movilidad motorizada (Cao et al., 2009; Cervero y Kockelman, 1997; Ewing y Cervero, 2001 y 2010; Næss, 2009), así como también desde la óptica de la movilidad peatonal (Ewing y Handy, 2009; Lee y Moudon, 2006). Trabajos que han estado generalmente encaminados a descifrar qué variables son más determinantes en este binomio.

Apoyado en gran medida sobre la producción de conocimiento en torno a la influencia de la estructura urbana en el patrón de viaje e inspirado en el planteamiento conceptual de Bertolini y Dijst (2003), el concepto entorno de movilidad se entiende como aquella unidad espacial operativa para la planificación y la evaluación de la movilidad urbana, resultante de una valoración integrada de factores de la estructura urbana y del patrón de viaje, capaz de aportar información sobre las siguientes cuatro dimensiones de la movilidad: urbanística, ambiental, socioeconómica y modal (Soria, 2011). Definición que se apoya sobre diversos trabajos que utilizan un enfoque conceptual próximo a éste, en lo que respecta al uso de unidades espaciales que vinculen factores de la estructura urbana y el patrón de viaje como matriz espacial sobre la que planificar la movilidad urbana, tanto motorizada como no motorizada.

Desde un enfoque orientado a la movilidad motorizada, Cervero (2002) centra parte de su investigación en la utilización de unidades espaciales para orientar la toma de decisiones en el campo de la movilidad. Tales unidades espaciales delimitan diferentes espacios urbanos en base a características homogéneas de densidad, diversidad y diseño, sobre las que se analiza la elección modal de transporte. Otro ejemplo relevante es el trabajo llevado a cabo por Delmelle y Casas (2012) en lo que respecta a la influencia de infraestructuras de transporte público sobre elementos de la estructura urbana.

Desde un enfoque orientado a la movilidad no motorizada y particularmente peatonal, la investigación llevada a cabo por Rodríguez et al. (2009) identifica distintas unidades urbanas, con el objetivo de generar medidas sinérgicas entre los desplazamientos peatonales y el sistema de autobús Transmilenio, en Bogotá (Colombia). Igualmente, cabe destacar otro conjunto de trabajos cuyo interés versa en conocer las características que condicionan una mayor o menor actividad peatonal en las áreas de influencia espacial del transporte público, con el objetivo de mejorar dichas características y, por ende, incrementar la influencia espacial de las paradas de transporte público (Olszewski y Wibowo, 2005; Weinstein et al., 2008). Este enfoque reconoce la importancia de analizar, a nivel urbano, aquellos factores que incentivan la movilidad no motorizada para que la implementación de distintos modos de transporte público tenga éxito, algo que ocurre, por ejemplo, en el caso de los autobuses públicos (Rodríguez et al., 2009) o en los sistemas de metro ligero (Hass-Klau y Crampton, 2002).

Centrando el discurso sobre la movilidad peatonal como enfoque dominante del artículo, cada vez es más frecuente usar la expresión entorno de movilidad peatonal o entorno peatonal (Zacharias, 2001) para denominar a aquellas unidades espaciales que se conforman a partir de la síntesis de factores urbanos vinculados estrechamente al desplazamiento peatonal y a modos alternativos de éste (Borst et al., 2009; Clifton et al., 2007). Tales entornos peatonales 
se caracterizan por tener una preferencia o una aptitud por el peatón como principal modo de desplazamiento, que deriva de poseer ciertas características favorables, tales como continuidad espacial, topografía o densidad urbana.

Profundizando sobre lo anterior, a la hora de evaluar o caracterizar entornos peatonales, es necesario considerar diversos factores de diseño urbano que influyen en el comportamiento del peatón a la hora de desplazarse (Borst et al., 2009; Lotfi y Koohsari, 2009; Zacharias, 2001), especialmente como consecuencia de la relación directa e intensa que el peatón mantiene con la ciudad a través de sus sentidos, lo que le permite interaccionar con otros peatones (Gehl, 1971; Peters, 1981), participar de la actividad comercial y cultural en las calles (Venturi et al., 1977) o apreciar su entorno natural y arquitectónico (Jacobs, 1993). En definitiva, la movilidad peatonal permite apreciar las características singulares de los itinerarios, lo cual facilita que cada uno tenga una identidad propia (Lynch, 1960).

A pesar de que se ha progresado en la identificación de entornos peatonales como instrumento para la planificación y se conoce mejor qué factores de la estructura urbana influyen en el desplazamiento peatonal (Ewing y Handy, 2009; Owen et al., 2004), es necesario avanzar en caracterizar y evaluar la calidad peatonal de tales entornos y en utilizar los métodos más idóneos para ello (Sauter et al., 2010).

\subsection{Factores determinantes de la calidad peatonal}

$\mathrm{Al}$ objeto de evaluar la calidad peatonal en un ámbito geográfico específico, es preciso no solo diseccionar la relación o las relaciones que se establecen entre los peatones y el entorno urbano por el que éstos transitan, sino también conocer qué condiciones propician o desincentivan la movilidad peatonal. Inicialmente, el estudio de los condicionantes de la movilidad peatonal cubrían aspectos como la seguridad, la conveniencia, la continuidad, el confort, la coherencia y el atractivo (Fruin, 1971). Aspectos que, con posterioridad, fueron reagrupados en los cuatro bloques siguientes: accesibilidad, seguridad, confort y atractivo (Alfonzo, 2005; Pozueta et al., 2009). Es por tanto que, en la medida en que tales condicionantes sean satisfechos, el entorno peatonal poseerá la calidad necesaria para que el peatón se desplace, lo que incidirá de manera decisiva en los niveles de servicio peatonal del entorno urbano (Olszewski y Wibowo, 2005). Tal aspecto es determinante si se trata, por ejemplo, de potenciar el acceso peatonal a determinadas centralidades urbanas, como podrían ser las estaciones de transporte público. De los cuatro factores citados anteriormente, la accesibilidad tiene un carácter eminentemente físico, mientras que los tres aspectos restantes poseen una mayor implicación perceptual. Además, dichos factores se encuentran vinculados mediante una relación secuencial (figura 1), en la que la accesibilidad es el aspecto condicionante que debe satisfacerse en primera instancia. El siguiente factor que deberíamos abordar sería la seguridad, para, posteriormente, poder emprender el factor del confort $y$, finalmente, el de atracción (Alfonzo, 2005). 


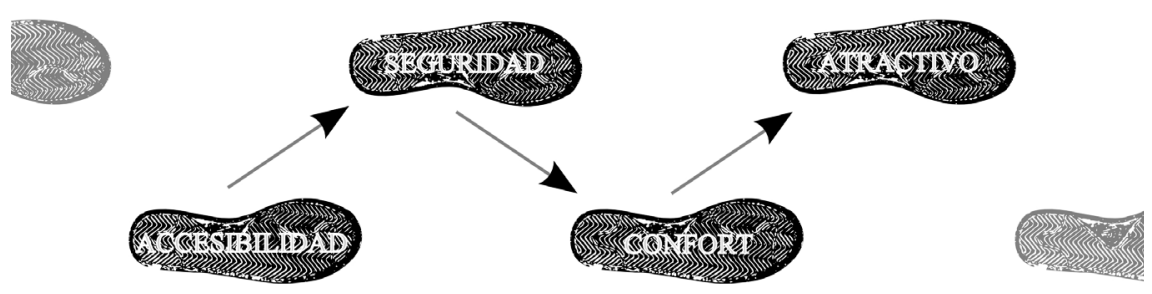

Figura 1. Aspectos condicionantes en la movilidad peatonal.

Fuente: elaboración propia a partir de Alfonzo (2005).

La accesibilidad, como condicionante estructurante y que está en el primer lugar de los aspectos previamente apuntados, hace referencia a aquellos aspectos más esenciales implicados en la movilidad peatonal. Es decir, aspectos relativos a la propia existencia de una infraestructura peatonal, la pendiente de ésta, su anchura o los materiales empleados para construirla. Así, por ejemplo, ciertas evidencias muestran que existe una relación entre la dimensión de la acera y la velocidad peatonal. Dicho de otra forma: una acera menor de dos metros de ancho puede originar que los encuentros entre peatones se den con dificultad (Prinz, 1986; Sanz, 2008), al ralentizar u obstruir el flujo peatonal. En este sentido, que la accesibilidad sea el primer condicionante estructural ha dado lugar a que sean numerosos los manuales de diseño que la tengan en consideración (Manchón et al., 1995; Portland, 1998; Pozueta, 2001; Sanz, 2008).

El segundo aspecto condicionante de la movilidad peatonal es la seguridad, en especial, la relacionada con el tráfico (Pikora et al., 2003). En esta línea, factores como la velocidad de circulación tiene claras repercusiones sobre la calidad de las personas que se desplazan a pie por la vía pública, ya que determina la sensación de seguridad que éstas tienen (Landis et al., 2001; Transport, 2007). Este aspecto se introduce en el diseño urbano mediante diferentes actuaciones destinadas a reducir la fricción entre modos de transporte, actuaciones como el templado del tráfico, la disminución de la velocidad o el espacio compartido, entre otras, y que, además, repercuten positivamente en la intensidad y en la diversidad de funciones urbanas (Sanz, 2008) y, por ende, en la calidad peatonal de una calle o de un ámbito determinado.

El confort es el tercero de los aspectos señalados como condicionantes de la movilidad peatonal y constituye, además, el aspecto cuya cuantificación puede suponer una mayor dificultad, por la diversidad de matices que puede contener. El análisis del confort como condicionante de la movilidad peatonal puede dividirse en tres ramas: físico, psicológico y fisiológico (Sarkar, 2003). En el seno de cada una de esas ramas, es posible encontrar multitud de variables vinculadas al confort, entre las que ocupan un papel destacado, por volumen de referencias, las variables climáticas (Nikolopoulou y Lykoudis, 2006; Stathopoulos et al., 2004). Además, estas variables pueden ser reguladas mediante elementos del diseño urbano, como, por ejemplo, el arbolado (Shashua-Bar y 
Hoffman, 2000), el cual permite crear sensación de encajonamiento en calles con amplias secciones, así como orientar el flujo peatonal, lo que ayuda a definir la vía, requisito indispensable para generar en el peatón la impresión de seguridad e intimidad (Peters, 1981). El movimiento de las hojas de los arboles (Jacobs, 1993) también le da a la calle un carácter especial. Desde una visión contrapuesta, en ocasiones, el confort para el peatón es analizado desde aquellas variables que generan estrés en el peatón y que generalmente están asociadas con el tráfico, como son el ruido (Raggam et al., 2007) y la polución.

Por último, la atracción hace referencia a los paisajes urbanos que originan itinerarios atractivos para los peatones. En este aspecto, desempeñan un papel fundamental las personas que van a pie, puesto que su movilidad les permite interaccionar entre ellas (Gehl, 1971; Peters, 1981) y, además, participan en la actividad comercial y cultural de las calles (Venturi et al., 1977). En esta línea, y de manera específica, los usos comerciales se configuran como puntos de atracción de gente que observa los distintos escaparates, que interactúa con otros individuos en estos establecimientos y que se deja inspirar (Gehl, 1971). Además, se establece una fuerte relación entre el diseño de la calle y los usos comerciales. Ello puede animar el comercio (Peters, 1981) e incluso crear, entre los distintos establecimientos, sinergias que fortalezcan su atracción (Salingaros et al., 2005). Por tanto, una calle densa en usos comerciales que, además, posea una diversidad de oferta resulta determinante para atraer peatones.

En base a la descripción realizada, resulta evidente profundizar en el desarrollo de métodos y de herramientas para evaluar la calidad del diseño urbano en la movilidad peatonal (Ewing y Handy, 2009). Es importante que dichos métodos se encuentren en una interfase entre la percepción de los peatones y los factores de diseño manejados por los planificadores. De esta forma, resulta necesario analizar las cualidades del diseño urbano que estén relacionadas con los aspectos condicionantes de la movilidad peatonal. No obstante, no todas las cualidades urbanas son cuantificables, dada su propia naturaleza. Entre las cualidades cuantificables destacan las cinco siguientes: escala humana, imagen evocadora, complejidad, confinamiento y trasparencia (Ewing y Handy, 2009), que serán valoradas en este artículo a través de indicadores que procuran «medir» las posibles relaciones entre las características físicas y el condicionamiento de la movilidad peatonal.

Así pues, establecer un conjunto reducido de indicadores cuya representatividad sea elevada a causa del número de relaciones que se establecen con diferentes cualidades del diseño urbano dota a los diferentes agentes de una herramienta útil, capaz de plasmar de forma técnica aquellos aspectos urbanos que condicionan una movilidad peatonal de calidad.

\section{Método para caracterizar la calidad peatonal de entornos de movilidad (CPEM)}

La metodología propuesta supone una herramienta útil si tenemos en cuenta dos aspectos: principalmente, por una parte, permite evaluar los entornos para 


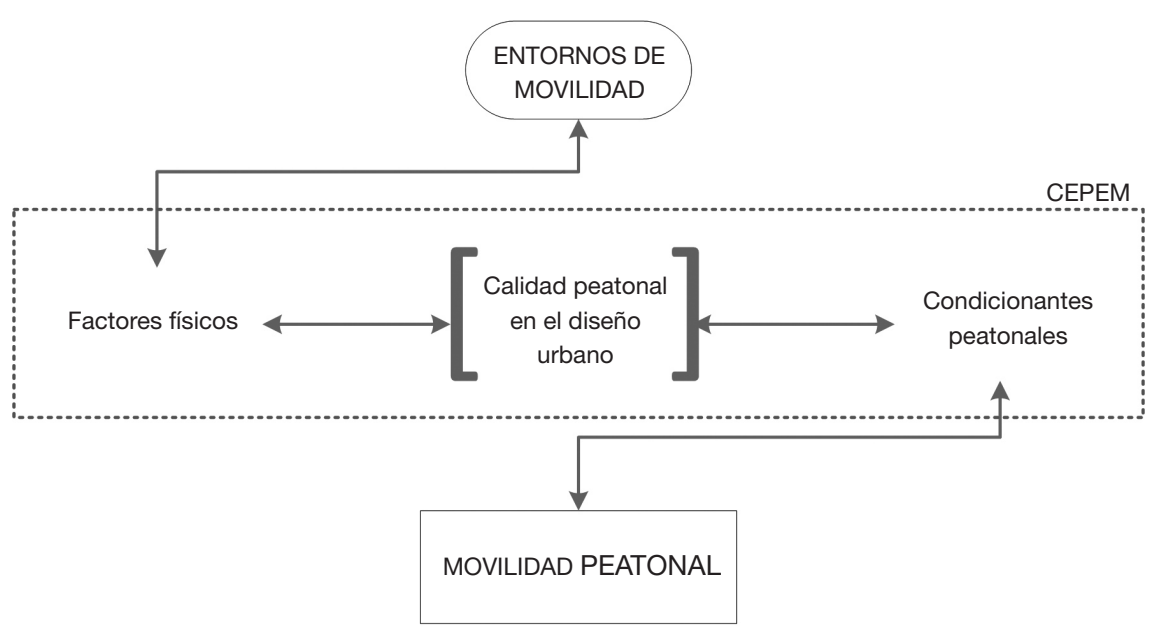

PLANIFICADORES

ACTORES

Figura 2. Metodología de caracterización peatonal de los entornos de movilidad (CPEM).

Fuente: elaboración propia.

la promoción de la movilidad peatonal y, por otra parte, como herramienta versátil que permite adaptarse a diferentes casos de estudio. Entrando en detalle en la metodología CPEM, ésta tiene como finalidad medir aquellas características presentes en los entornos de movilidad y su influencia sobre el desplazamiento peatonal. En dicho sentido, la presencia de características físicas y de calidad en los entornos de movilidad no sólo satisfará los condicionantes peatonales, sino que, lógicamente, favorecerá el desplazamiento de las personas que van a pie por dichos entornos.

La aplicación del método CPEM consta de tres fases: selección de indicadores, estandarización o normalización de resultados y aplicación del caso de estudio.

\subsection{Fase I. Selección de indicadores}

La caracterización peatonal de los entornos de movilidad precisa contar con una serie de indicadores capaces de cuantificar aquellas características físicas, relacionadas con las principales cualidades del diseño urbano, que forman parte de los condicionantes del desplazamiento de las personas que van a pie por la vía pública. Estos indicadores están basados en una serie de criterios de buenas prácticas para la selección de indicadores, entre los cuales se encuentran la representatividad, la facilidad de aplicación y la comprensión (Litman, 2012). Representatividad para reducir el número de indicadores lo máximo posible, sin que con ello pierda validez el análisis de las cualidades 
de diseño urbano que repercuten en los aspectos condicionantes. Facilidad de aplicación para que la información que se deba introducir esté disponible en las administraciones o sean sencillas de adquirir, así como facilidad de manejo del indicador que permita ser aplicado por los diferentes actores. Por último, los indicadores deben ser comprensibles por parte de cualquier actor que participe en el proceso, sin que sean necesarios conocimientos técnicos o complejos en la materia.

De acuerdo con los criterios de representatividad, facilidad de aplicación y comprensión mencionados anteriormente, se establecen una serie de indicadores para la caracterización peatonal de los entornos de movilidad. Indicadores que son, además, representativos de una serie de cualidades deseables, entre las que se encuentran aquellas que permiten su cuantificación, como el confinamiento, la escala humana, la complejidad y la imagen evocadora, y que aportan calidad al entorno desde el punto de vista de la movilidad peatonal. Los indicadores utilizados corresponden a una adaptación de algunas de las medidas operativas ya planteadas por Ewing et al. (2005) o Pikora et al. (2006) y que se detallan a continuación. También se recogen de manera sintética en la tabla 2.

Para el primer condicionante de la movilidad peatonal, la accesibilidad, se ha utilizado el indicador de sección peatonal, dada la facilidad de concepción y lo básico y primario que resulta para que la movilidad peatonal tenga lugar, al modular los flujos de las personas que se desplazan a pie por la vía pública (Prinz, 1986; Sanz, 2008). Este indicador permite conocer en qué lugares del entorno de movilidad los flujos peatonales se desarrollan sin problemas, dadas las dimensiones de la sección peatonal, y donde los flujos peatonales podrían ralentizarse e incluso entrar en conflicto con otros modos de transporte, debido a una sección peatonal insuficiente.

El indicador utilizado en el segundo de los aspectos, relativo a seguridad vial, es la fricción modal. Este indicador considera la velocidad máxima permitida en la vía, así como el número de carriles de transporte motorizado que posee, de tal modo que, a mayor velocidad y mayor número de carriles, más elevadas serán la fricción existente y la percepción de inseguridad que posea el peatón respecto a estas zonas (Landis et al., 2001).

El confort, como aspecto condicionante, es uno de los que requieren un mayor número de indicadores en su valoración, dada la diversidad de factores del diseño urbano que intervienen en la generación de confort para el peatón (Sarkar, 2003). Además, estos factores poseen un grado más elevado de independencia, por lo que difícilmente pueden ser relacionados a través de un único indicador. En este sentido, se han seleccionado los indicadores de densidad de arbolado, ruido y ratio entre la anchura y la altura de la calle. Pasamos a comentarlos.

La densidad de arbolado permite, como indicador, establecer relaciones con otras características de confort, como es la generación de sombra, la regulación de temperatura y de humedad, así como una renaturalización de las calles. Este indicador, además, permite evaluar cualidades del diseño urbano, 


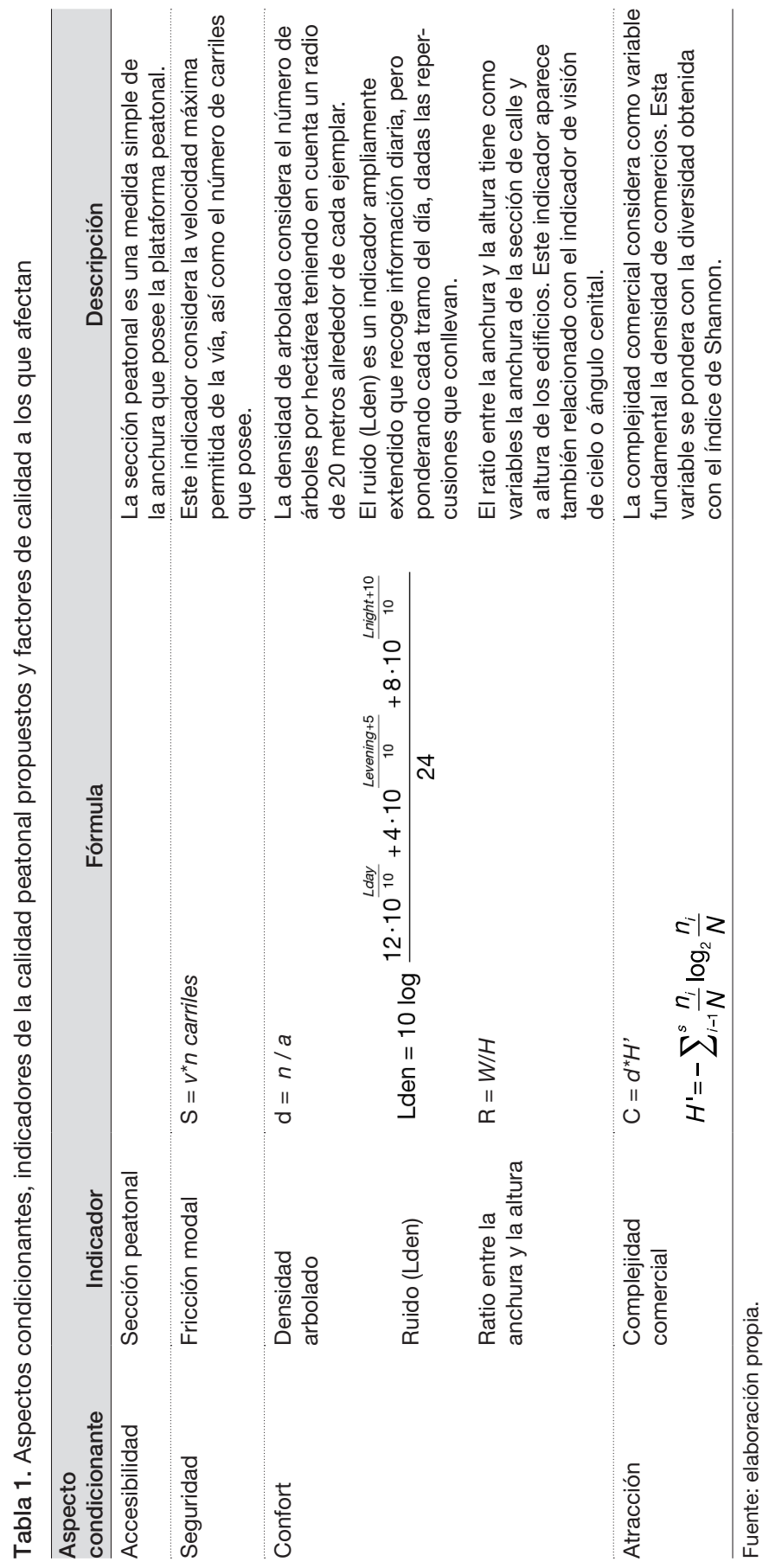


como la generación de imágenes evocadoras (Jacobs, 1993), la complejidad y la mejora del confinamiento.

En lo referente al ruido (Lden), este indicador posibilita analizar otro de los factores determinantes en el confort (Raggam et al., 2007). Es un factor muy tenido en cuenta cuando se habla de movilidad y de calidad ambiental urbana. Además, el ruido constituye uno de los indicadores más relevantes en lo que respecta a la generación de imágenes evocadoras (Ewing et al., 2005).

Finalmente, el indicador de la ratio existente entre la anchura y la altura tiene una marcada relevancia, porque establece múltiples relaciones, tanto con otros indicadores de confort como con otros condicionantes, como la seguridad. Así mismo, posee relación con varias cualidades deseables, como la generación de confinamiento positivo o la escala humana (Jacobs, 1993). Por tanto, usar la ratio que relaciona la anchura con la altura supone evaluar la influencia de las variables más básicas en la planificación urbanística, como son altura de edificios y sección de la calle en la generación de confort, tanto a nivel psicológico como a nivel ambiental, al estar relacionado con la insolación que recibe la calle (Alitoudert y Mayer, 2006).

Por último, en cuanto al atractivo como condicionante de la movilidad peatonal, se propone el indicador de complejidad comercial, entendiendo éste como una síntesis de la densidad y la diversidad comercial. Además, como su propio nombre indica, la complejidad comercial está relacionada con la complejidad como cualidad de diseño urbano, ya que en ellas se incluyen actividades como restaurantes y bares o comercios con amplios escaparates que resultan atractivos para el peatón. En este sentido, el indicador permite evaluar las principales actividades generadoras de interacciones potenciales entre personas y que son, en consecuencia, generadoras de atracción para los peatones (Carmona, 2003).

\subsection{Fase II. Estandarización de resultados}

Las ventajas de estandarizar los resultados obtenidos son varias. Por una parte, se establecen unos umbrales de calidad peatonal sobre factores en los que, en términos generales, no existe una tradición reguladora al respecto. Por otra parte, el establecimiento de tales umbrales de calidad peatonal contribuirá a desarrollar métodos exportables a otros contextos y regiones (Soria, 2011), así como a generar una concienciación ambiental.

Además, la estandarización de los resultados de cada uno de los indicadores propuestos permite obtener una información sobre la calidad de cada factor que puede ser fácilmente comparable y entendible por parte de los diferentes actores. Bajo este contexto, se lleva a cabo una estandarización de los valores en función de la diversa bibliografía científica existente al respecto, manuales de diseño urbano y buenas prácticas. Esta estandarización se compone de cinco categorías o niveles de calidad (tabla 2), que son resultado de las características presentes en las calles del entorno de movilidad analizado. 
Tabla 2. Estandarización de los resultados en niveles de calidad peatonal

\begin{tabular}{ccccccc}
\hline $\begin{array}{c}\text { Nivel de } \\
\text { calidad } \\
\text { peatonal }\end{array}$ & $\begin{array}{c}\text { Sección } \\
\text { peatonal } \\
(\mathrm{m})\end{array}$ & $\begin{array}{c}\text { Fricción modal } \\
\text { Velocidad }(\mathrm{km} / \mathrm{h})\end{array}$ & $\begin{array}{c}\text { Ruido } \\
\mathrm{y} \text { carriles }\end{array}$ & $\begin{array}{c}\text { Densidad de }(\mathrm{dBA}) \\
\text { arbolado } \\
\text { (arb./km²) }\end{array}$ & $\begin{array}{c}\text { Ratio entre la anchura } \\
\text { y la altura } \\
\text { Anch/Alt }\end{array}$ & $\begin{array}{c}\text { Complejidad } \\
\text { comercial } \\
\text { (comercios/ha) }\end{array}$ \\
\hline I & \multicolumn{1}{c}{ (1) } & $(2)$ & $(3)$ & $(4)$ & $(5)$ & $(6)$ \\
II & $3-1,8$ & Peatonal & $<60$ & $>10.000$ & $1: 2-1: 3$ & $\geq 64$ \\
III & $1,8-1,2$ & 50 y 1 carril & $65-70$ & $2.500-1.000$ & $3: 2-3: 1 / 1: 3: 1: 4$ & $25-40$ \\
IV & $1,2-0,9$ & 50 y 2 carriles & $70-75$ & $1.000-650$ & $>3: 1^{*}$ & $9-25$ \\
V & $<0,9$ & 50 y $\geq 3$ carriles & $>75$ & $<650$ & $<1: 4$ & $<9$ \\
\hline
\end{tabular}

* Se considera que posee un mayor nivel de calidad peatonal por la potencial sinergia con el arbolado que mejoraría la percepción espacial.

Los valores indicados en la tabla se establecen a partir de la interpretación de la información contenida en las siguientes referencias.

1. Manchón et al. (1995); Prinz (1986); Sanz (2008)

2. Sanz (2008)

3. EEA (2010)

4. Manchón et al. (1995)

5. Alitoudert y Mayer (2006); Bentley (1999); Jacobs (1993); Pozueta Echavarri et al. (2009)

6. Intervalos obtenidos mediante cuantiles

Fuente: elaboración propia a partir de las referencias citadas.

\subsection{Fase III. Aplicación al caso de estudio. Entornos de movilidad en el área metropolitana de Granada}

El método de caracterización peatonal de entornos de movilidad (CPEM) se caracteriza por ser flexible, lo que facilita su adaptación y su aplicación a diferentes casos de estudio según las necesidades específicas. De esta forma, el método puede ser aplicado en diversas escalas y con diferentes enfoques (más técnicos o más orientados a la decisión).

Como caso práctico para este artículo, se ha seleccionado un corredor de movilidad en el área metropolitana de Granada (España). Durante los últimos treinta años, el proceso de metropolitanización en la comarca de la Vega de Granada ha reforzado una espiral de interdependencia entre los municipios que la conforman (Aguilera, 2008), en parte traducidos en flujos de viajes crecientes, especialmente en el sentido que va de la corona de municipios a la capital. A pesar de la creación del Consorcio de Transportes Metropolitanos y la consecuente Red Integrada de Transporte Público, problemas como el crecimiento del número de automóviles y la congestión consiguiente suponen una amenaza para la calidad del aire local, la contaminación acústica, la deslocalización territorial de usos del suelo, etc.

Ante este panorama, las instituciones competentes han optado por implantar un sistema de metro ligero en el principal corredor de movilidad de la región, utilizándolo como instrumento impulsor de nuevas pautas de movilidad. Contexto en el que destaca la voluntad de la corporación municipal y el gobierno regional por convertir al metro ligero en un factor de atracción peatonal en los entornos de sus estaciones. El corredor lo 
Tabla 3. Caracterización de los entornos de movilidad analizados

\begin{tabular}{lcc}
\hline & $\begin{array}{c}\text { Entorno de circulación } \\
\text { motorizada }\end{array}$ & $\begin{array}{c}\text { Entorno de proximidad } \\
\text { y alcance local }\end{array}$ \\
\hline $\begin{array}{l}\text { Densidad residencial } \\
\text { (viviendas/ha) }\end{array}$ & $<30$ & $\geq 95$ \\
$\begin{array}{l}\text { Intensidad de actividades } \\
\text { (actividades/1.000 viviendas) }\end{array}$ & 53,56 & 100 \\
$\begin{array}{l}\text { Tránsito motorizado } \\
\text { (vehículos/actividad) }\end{array}$ & & \\
$\quad$ (autobuses/actividad) & 384,27 & 46,02 \\
& 23,68 & 4,30 \\
\hline
\end{tabular}

Fuente: elaboración propia.

conforman cuatro municipios: Albolote, Armilla, Granada y Maracena (ver figura 3).

En este contexto, la Universidad de Granada recibe el encargo ${ }^{1}$ de estudiar diferentes ámbitos del corredor, con el fin de evaluar su calidad desde el punto de vista de su movilidad peatonal, como paso previo a la definición de posibles estrategias urbanas, priorización de inversiones económicas, etc. En respuesta a esta demanda, desde la Universidad, se identifican diferentes entornos de movilidad en el corredor y se reconocen diferentes aptitudes peatonales en cada uno de ellos. Sobre tales entornos de movilidad, se decide aplicar el método de CPEM para caracterizar, de una forma precisa, su calidad peatonal y se toman sus resultados como base para orientar a la Administración pública en la toma de decisiones.

Utilizando diferentes indicadores vinculados a la estructura de la ciudad (densidad residencial, diversidad de actividades urbanas, cobertura temporal de actividades urbanas, etc.) y del patrón de viaje (tasas de circulación motorizada, influencia de ejes transversales sobre el corredor, etc.), fueron identificados hasta cinco entornos de movilidad:

1. Entorno de proximidad y alcance local.

2. Entorno de proximidad y distribución circulatoria.

3. Entorno de circulación motorizada.

4. Entorno de estaciones intermodales.

5. Entorno de centralidad metropolitana.

Específicamente, son presentados aquí los resultados obtenidos para el entorno de proximidad y alcance local y el entorno de circulación motorizada (tabla 3), ya que constituyen los entornos de movilidad sobre los que se aplicará el método CPEM. El motivo es que, inicialmente, eran entornos de movilidad en el corredor, con características urbanas y patrones de viaje

1. Contrato de investigación con la empresa Ferrocarriles de Andalucía (2009-2011): El metropolitano de Granada como instrumento de innovación, calidad y sostenibilidad urbana. 


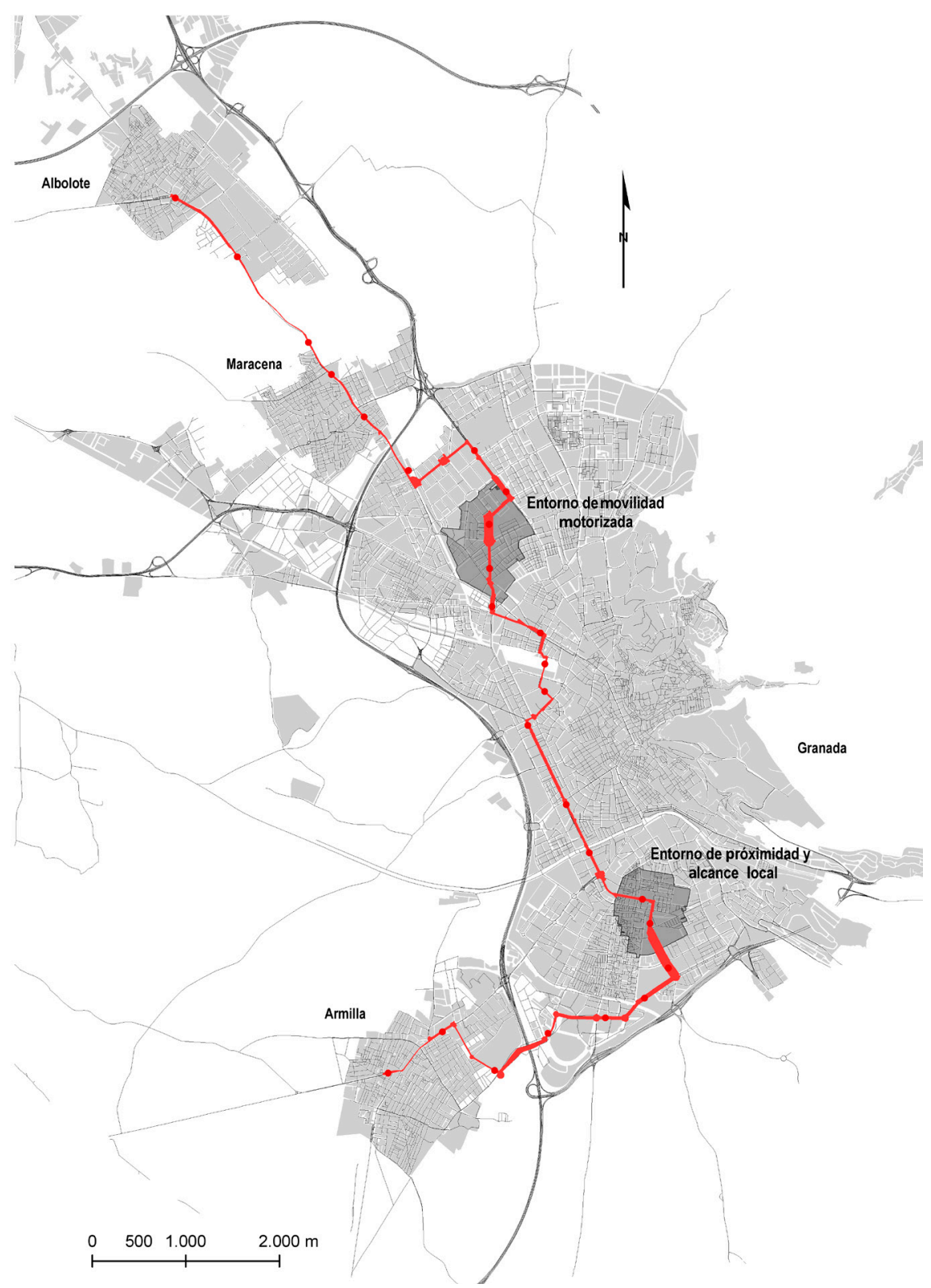

Figura 3. Entornos de movilidad seleccionados para aplicar el método CPEM.

Fuente: elaboración propia. 
sustancialmente diferentes. Mientras que el entorno de proximidad y alcance local mostraba una alta aptitud inicial al desplazamiento peatonal, ocurría todo lo contrario en el entorno de circulación motorizada. De ahí que se consideraran los dos entornos de movilidad más idóneos para aplicar el modelo CPEM y testear su sensibilidad y su capacidad para caracterizar la calidad peatonal de tales entornos.

El entorno de proximidad y alcance local hace referencia a aquellos tramos del corredor cuya movilidad está caracterizada por una fuerte dimensión local del medio urbano y, por lo tanto, la mayoría de sus flujos de movilidad deberían encontrarse altamente mediatizados por demandas locales del entorno inmediato del corredor. La componente de proximidad es muy relevante, por lo que una gran parte de las necesidades de la población residente podrían ser cubiertas sin tener que utilizar modos motorizados. De ahí que no sean espacios que destaquen por ser centros de atracción de movilidad a nivel metropolitano, como tampoco son espacios urbanos caracterizados por un alto tránsito, a lo que contribuye el hecho de que no estén conectados a ejes transversales de relevancia, ni desde el punto de vista de su movilidad motorizada ni no motorizada.

El entorno de movilidad motorizada hace referencia a aquellos tramos cuya circulación está caracterizada por una muy débil dimensión local del medio urbano, aspecto que refuerza su condición como lugares de tránsito y/o desplazamientos mediante vehículos. De esta forma, la componente de proximidad es poco relevante en la mayoría de casos, por lo que una gran parte de las necesidades de la población residente no pueden ser satisfechas en ese mismo espacio. De ahí que, desde el punto de vista de la movilidad, se conviertan en lugares destinados exclusivamente al tránsito o a la circulación motorizada de unos lugares a otros del corredor, o entre el corredor y el resto del sistema urbano, situación que, en muchos casos, se ve reforzada por su función como itinerarios circulatorios en el diseño de la propia ciudad.

\section{Aplicación del método CPEM y resultados obtenidos}

Una vez caracterizados los entornos de movilidad, la aplicación del método CPEM permite obtener, de manera detallada, las implicaciones de cada uno de los entornos descritos respecto a la calidad peatonal, a través de la evaluación de los aspectos que condicionan la movilidad de las personas que se desplazan a pie. En este sentido, a continuación, se detallan los principales resultados obtenidos.

\subsection{Accesibilidad}

La calidad peatonal respecto al indicador de sección peatonal (imagen $a$, figura 4) muestra resultados similares en los dos entornos seleccionados, en los cuales existe una superficie superior al $80 \%$ del total, con una presencia de aceras superiores a 3 metros de sección. Además, ambos ámbitos comparten 


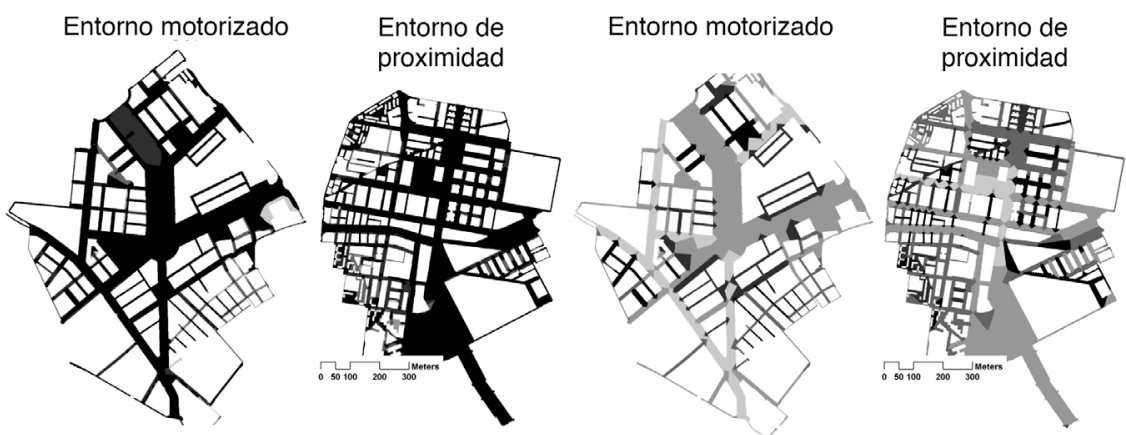

a) Sección peatonal Nivel de calidad $\quad$ b) Fricción modal
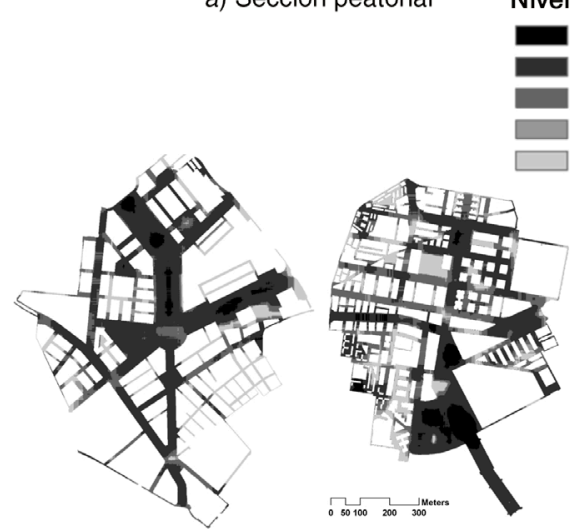

1 - Alto

c) Densidad de arbolado

Nivel de calidad

$$
\begin{aligned}
& 1 \text { - Alto } \\
& 2 \\
& 3 \\
& \square \\
& \square \text { - Bajo }
\end{aligned}
$$

\section{5 - Bajo}
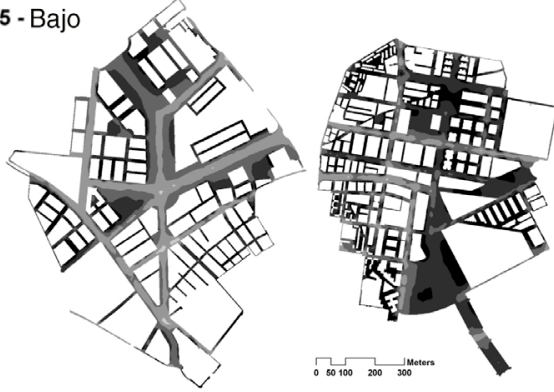

d) Ruido

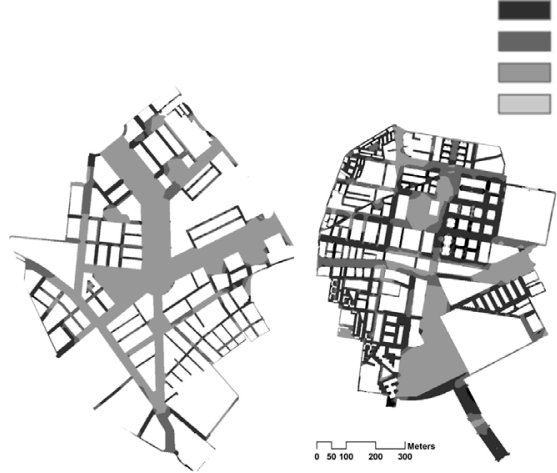

e) Ratio entre la anchura y la altura

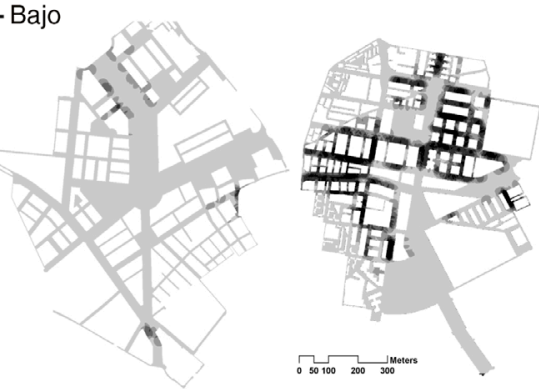

f) Complejidad comercial

Figura 4. Niveles de calidad según los indicadores de los entornos analizados: motorizado y de proximidad.

Fuente: elaboración propia. 
características similares en cuanto a la distribución de las diferentes secciones peatonales. Las mayores secciones peatonales se encuentran en las avenidas o calles principales que articulan el entorno, donde se sitúan las paradas de metro ligero. Por tanto, ambos entornos presentan un entramado de calles cuya sección peatonal permite al peatón desplazarse cómodamente, a priori, sin interferencias de obstáculos. En efecto, la sección peatonal existente permite compatibilizar la sección mínima requerida para un flujo peatonal óptimo y la presencia de mobiliario urbano, del mismo modo que permite la presencia de peatones parados frente a escaparates comerciales en caso de que los haya, sin interferir en el resto de personas que se desplazan a pie (Manchón et al., 1995; Sanz, 2008).

\subsection{Seguridad}

La fricción modal como indicador estructural de la seguridad vial para el peatón y, por tanto, la seguridad que ofrecen las distintas calles a la hora de emprender un desplazamiento peatonal muestra, en primer lugar, que, en ambos entornos, el grueso de la superficie se encuentra en niveles de calidad 3 (entre el $44 \%$ y el $49 \%$ ), nivel que está definido por la presencia de calles con límite de velocidad de $50 \mathrm{~km} / \mathrm{h}$ y dos carriles de circulación. Entre las calles con estas características, se encuentran aquellas por las que pasa la línea de metro ligero, que mantienen y no disminuyen el nivel de fricción modal, al decidirse no alterar el número de carriles de tráfico existentes. Por otra parte, en lo que respecta a la distribución de superficie en los niveles de calidad restantes, se observan diferencias claras según el entorno analizado (imagen b, figura 4). En este sentido, la superficie peatonal es mayor en el entorno de proximidad que en el entorno de movilidad motorizado, lo que da lugar a un mayor porcentaje de nivel 1 de calidad (12\%), mientras que, en el nivel 2, destaca el entorno de circulación motorizada, debido a la mayor presencia (15,5\% de la superficie) de zonas 30. Por otra parte, el entorno de circulación motorizada presenta, como rasgo más significativo, un porcentaje notable de superficie de calles $(21,1 \%)$ que cuentan con tres o más carriles para el tráfico motorizado y con un límite de velocidad de $50 \mathrm{~km} / \mathrm{h}$.

\subsection{Confort}

El indicador ruido (Lden) como evaluador de la calidad peatonal muestra, como se aprecia en la imagen comparada (imagen d, figura 4), niveles más elevados de calidad para el peatón en el entorno de proximidad y alcance local que en el entorno de circulación motorizada. En este sentido, más del $70 \%$ de la superficie libre del entorno de proximidad se encuentra en los dos niveles superiores de calidad peatonal respecto al ruido, es decir, tiene valores de Lden inferiores a los $65 \mathrm{dBA}$. En cambio, para el entorno de circulación motorizada, la superficie con calidades i y ii se reduce hasta el 37\% de la superficie libre total. Por tanto, este indicador ratifica, según los resultados obtenidos, la defi- 
nición de los propios entornos. El entorno de circulación motorizada presenta, en relación con el peatón, una menor calidad, puesto que los niveles de ruido existentes tienen como consecuencia un bajo confort acústico que puede condicionar la movilidad peatonal. Finalmente, y en contraposición, el entorno de proximidad y alcance local presenta unas características de ruido que resultan más confortables para el peatón a la hora de emprender un desplazamiento, a lo que se añade la imagen positiva que las calles de este entorno suscitan al peatón.

Por su parte, la densidad de arbolado, como indicador de cualidades urbanas como la imagen o la escala humana, muestra que las superficies con nivel de calidad 1 (imagen $c$, figura 4), con densidad de arbolado $>10.000$ unidades por kilómetro cuadrado, son reducidas, tanto en el entorno de proximidad y alcance local, como en el entorno de circulación motorizada. No obstante, en lo que a arbolado respecta, se hacen perceptibles diferencias considerables en la superficie de los entornos en referencia al nivel 2 de calidad peatonal, de modo que resulta superior en el entorno de circulación motorizada $(60,43 \%)$ que en el entorno de proximidad (44,25\%). Esta diferencia en la superficie de espacio libre con un nivel 2 de calidad se explica, como se puede apreciar en la imagen $c$ (figura 4), porque, en el entorno de circulación motorizada, hay bulevares con una alta densidad de arbolado, espacios que son sustancialmente menores en el entorno de proximidad.

En lo referente al indicador de ratio entre anchura y altura, los ámbitos presentan características diferentes (imagen $e$, figura 4). El entorno de circulación motorizada presenta una concentración de superficie superior al 64\%, con un nivel de calidad peatonal 4, lo que evidencia la presencia de calles y avenidas en las que la altura de la edificación es baja en relación con la sección de calle. El entorno de proximidad y alcance local presenta una distribución de la superficie más o menos homogénea en los niveles centrales (es decir, 2, 3 y 4). Sin embargo, es preciso matizar aquellos valores correspondientes al nivel 4 referentes a la ratio entre anchura y altura superior a 3 , debido a que éstos corresponden, prácticamente en su totalidad, a espacios públicos y zonas verdes.

Por tanto, comparando ambos entornos en lo que respecta a su calidad peatonal medida en función de la ratio resultante entre anchura y altura, se puede concluir que el entorno de proximidad y alcance local presenta un entramado de calles con una relación entre la sección y la altura de los edificios que confiere al peatón mayor sensación de confort.

\subsection{Atractivo}

El último indicador, el que se refiere a la complejidad comercial, muestra diferencias notables entre los entornos analizados (imagen $f$, figura 4 ), diferencias determinadas tanto por la densidad de usos comerciales que se encuentran presentes en el entorno de proximidad y alcance local, como por la diversidad de los mismos. Este hecho otorga a las calles un carácter atrayente, lo que facilita, pues, la movilidad peatonal, así como el nivel peatonal de servicio de las paradas de metro ligero. 

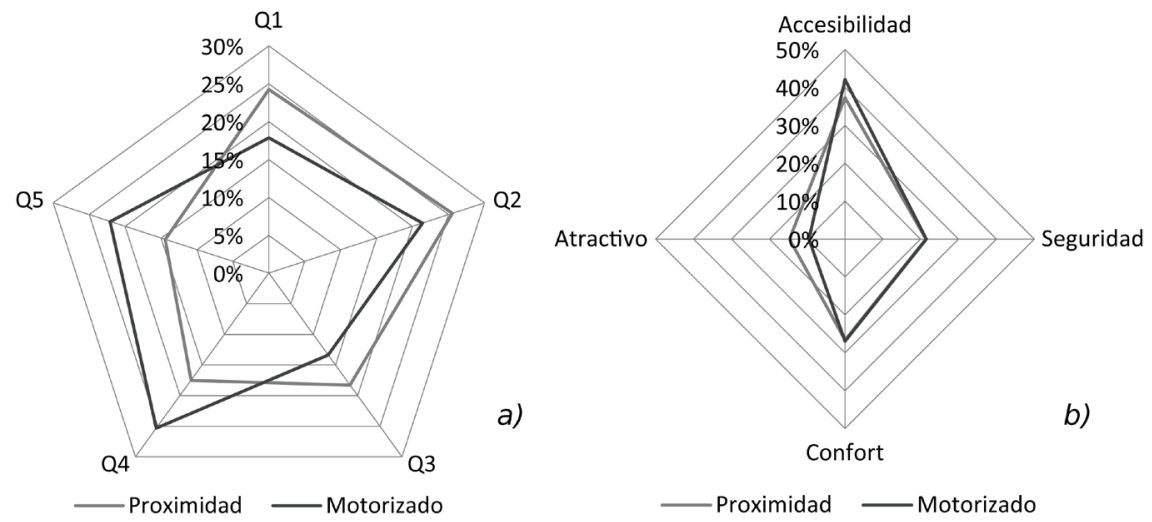

Figura 5. Porcentajes medios en los niveles de calidad peatonal a) y distribución según condicionantes $b$ ) para los entornos estudiados.

Fuente: elaboración propia.

En este sentido, cabe mencionar, en primer lugar, como el nivel 5 de calidad posee unos elevados porcentajes (un $62,8 \%$ por lo que respecta al entorno de proximidad y un $94,2 \%$ por lo que respecta al entorno de circulación motorizada), por la presencia de espacios públicos de naturaleza distinta y unido a las propias características de la herramienta de análisis utilizada, que establece un radio de búsqueda de 20 metros. Además, las diferencias entre los entornos en los primeros niveles de calidad establecidos para el indicador de densidad y diversidad son significativas, ya que, en el entorno de circulación motorizada, los porcentajes de superficie para estos niveles de calidad son prácticamente inexistentes (inferiores al 1\%).

Por otra parte, y en sentido más general, los resultados de calidad peatonal obtenidos para cada uno de los indicadores analizados muestran valores medios de porcentajes de calidad (imagen $a$, figura 5), sustancialmente diferentes entre los entornos de movilidad estudiados. Así pues, el entorno de proximidad posee unos valores medios de nivel de calidad 1, 2 y 3 superiores al entorno de circulación motorizada, entre los que destaca este último, puesto que presenta mayores porcentajes medios en los niveles de calidad 4 y 5 .

Desde otro punto de vista, analizar los resultados obtenidos de los niveles de calidad en función de los aspectos condicionantes de la movilidad peatonal para los entornos de movilidad estudiados permite observar dónde se centran los esfuerzos para satisfacer los condicionantes peatonales. Así pues, en ambos entornos, se observa (imagen $b$, figura 5) que existe prácticamente la misma distribución para satisfacer el condicionante de la accesibilidad, seguido de los condicionantes de seguridad y confort y, en último lugar, el condicionante de atracción. Sin embargo, las diferencias más notables, como se puede apreciar a modo de ejemplo en la figura 7 , se encuentran en el condicionante de atracción, para el que el entorno de proximidad presenta valores más elevados. 


\section{Entorno de movilidad motorizada}

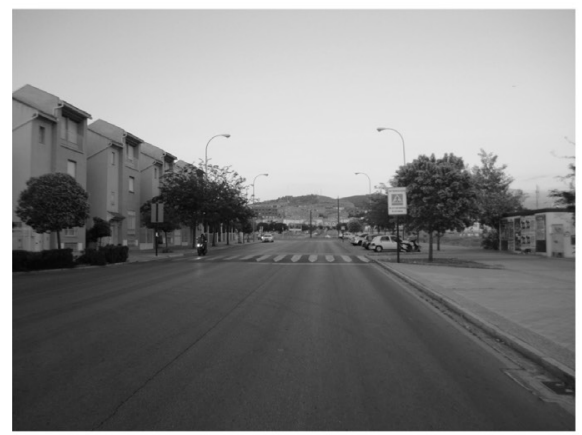

Entorno de proximidad y alcance local

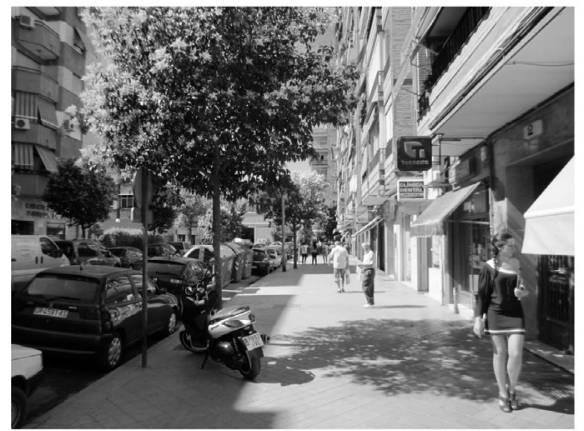

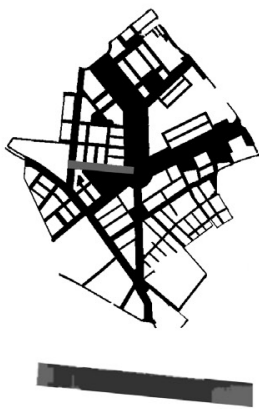

c)

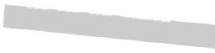

e)
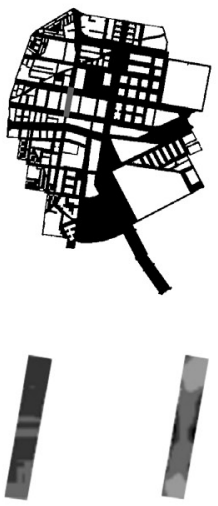

c)

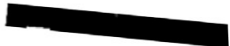

a)

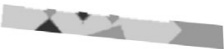

b)

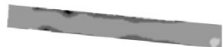

d)

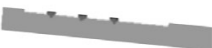

f)

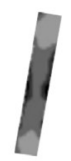

d)

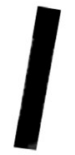

a)

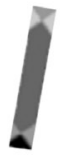

b)

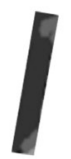

e)

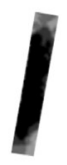

f)

Nivel de calidad

\begin{tabular}{|c|c|c|c|}
\hline $\begin{array}{l}1 \text { - Alto } \\
2\end{array}$ & a) Sección peatonal & b) Fricción modal & c) Densidad de arbolado \\
\hline 3 & & & \\
\hline $\begin{array}{l}4 \\
5 \text { - Bajo }\end{array}$ & d) Ruido & $\begin{array}{l}\text { e) Ratio entre la anchura } \\
\text { y la altura }\end{array}$ & f) Complejidad comercial \\
\hline
\end{tabular}

Figura 6. Ejemplo de niveles de calidad obtenidos para varias calles de los entornos de movilidad evaluados.

Fuente: elaboración propia. 


\section{Discusión y conclusiones}

El presente artículo ha profundizado sobre el desarrollo de instrumentos y herramientas metodológicos que faciliten la caracterización y la evaluación de entornos de movilidad desde el punto de vista de su calidad peatonal. Para ello, ha sido propuesto el método CPEM (caracterización peatonal de entornos de movilidad). Dicho método fue aplicado al caso práctico de dos entornos de movilidad en un corredor en el área metropolitana de Granada (España), donde las instituciones competentes decidieron implantar un sistema de metro ligero como factor de atracción peatonal, entre otros objetivos.

También se pueden obtener apreciaciones diferentes como resultado de aplicar el método CPEM. Algunas de sus ventajas más aparentes están vinculadas a su sencillez y flexibilidad, lo que influye indudablemente en el nivel de exportabilidad que presentan. El método es sencillo de aplicar, fundamentalmente porque no requiere de la utilización de ningún paquete específico de programas más allá del software GIS, comúnmente utilizado en el sector académico y profesional. Tampoco se ha precisado de la utilización de técnicas estadísticas complejas para entender los resultados obtenidos. Estas son grandes ventajas, ya que se facilita enormemente su uso potencial en el ámbito profesional. Tanto el modelo conceptual utilizado en su diseño, como las distintas fases que conforman el modelo CEPM convierten a éste en una herramienta metodológica capaz de operar en ámbitos escalares, competenciales y contextuales diferentes. Puede ser de utilidad tanto en sistemas de planificación donde la componente técnica tiene un papel destacado, como en sistemas de planificación más comunicativos y donde los resultados del método podrían ser entendidos como una guía para la toma de decisiones. Su flexibilidad se fortalece en la medida en que el método CEPM ha sido concebido como un modelo de caja blanca, donde sus posibles usuarios pueden decidir sobre el tipo de indicadores que se utilizan, el nivel de interacción de éstos, etc.

En contrapunto, es necesario señalar también algunos aspectos de progreso y mejora. A pesar de que los resultados del método nos han proporcionado interesantes lecturas parciales de cada uno de los cuatro bloques que lo conforman a nivel conceptual (accesibilidad, seguridad, confort y atracción), la obtención de un valor agregado o de conjunto puede contribuir a reforzar la utilidad del método CPEM en la toma de decisiones. Esencialmente porque sintetizar la caracterización de la calidad peatonal en un único valor final posibilita la comparación entre entornos de movilidad de forma ágil y clara, permite acercar la evaluación realizada a técnicos que no son especialistas en el campo, pero que necesariamente intervienen en la toma de decisiones, y reduce posibles barreras comunicativas entre los diferentes campos disciplinares que se dan cita en el proceso de planificación de la movilidad urbana. De igual manera, fortalecer el método propuesto con estudios preliminares, que ayuden a establecer qué variables influyen más en la calidad peatonal en función del contexto específico donde el modelo CPEM es aplicado, contribuiría a reducir la incertidumbre 
sobre la conveniencia de los indicadores utilizados o sobre el peso específico que debe tener en la evaluación cada uno de los cuatro bloques parciales apuntados. Esta cuestión es importante, porque la componente subjetiva desempeña un papel destacado a la hora de valorar la calidad peatonal, tal y como se ha señalado en diferentes lugares del artículo. Algunos procedimientos que pueden ayudar en este sentido son la elaboración de cuestionarios, las técnicas Delphi, etc. que permiten obtener valores de influencia para cada uno de los factores. Una vez conseguidos dichos valores, sería posible obtener el peso que cada factor tiene en el valor de referencia que la población o los expertos asignan a la distancia como medida tradicional de la accesibilidad. Con esta ponderación de factores, se puede obtener un valor unitario de calidad peatonal, así como establecer los niveles de servicio de cada una de las paradas de metro ligero en función de la calidad peatonal.

La selección de dos entornos de movilidad para aplicar el método CPEM se debe, esencialmente, al hecho que, a priori, tales entornos (entorno de proximidad y alcance local y entorno de circulación motorizada) mostraban aptitudes diferentes respecto de la movilidad peatonal. Consecuentemente, esto debería influir en la implementación del sistema de metro ligero en el corredor en cuestión. Esta diversidad los hacía idóneos para deducir si el método propuesto en este artículo era suficientemente sensible para detectar las diferencias que, a priori, se intuían y analizarlas con el fin de desarrollar estrategias eventuales de planificación. A la vista de los resultados obtenidos, se puede decir que el método ha sido sensible a las características que, de inicio, se presuponían para ambos entornos de movilidad. El método revela algunos aspectos interesantes que permiten alcanzar un conocimiento más profundo de estos espacios, de cara a plantear su utilidad desde el punto de vista de la atracción peatonal en torno al nuevo sistema de metro ligero.

En esta línea, los resultados obtenidos para los entornos de movilidad permiten, además, evidenciar aspectos susceptibles de mejora a través de actuaciones diferentes. Estas mejoras estarían en relación con los distintos planteamientos estratégicos de movilidad que se quieran llevar a cabo en los entornos. Estrategias como continuidad peatonal, articulación de espacios verdes, etc., que den mayor integridad a los entornos de movilidad.

Finalmente, hay que señalar que el artículo abre diferentes líneas de trabajo sobre las que sus autores ya se encuentran ocupados actualmente. Por un lado, optimizar el modelo CPEM, y su aplicación es una de las prioridades principales que surgen tras esta investigación. Dicha optimización pasa por integrar la opinión de las personas que van a pie por la vía pública sobre los factores condicionantes de la calidad peatonal en cada contexto. También hay que progresar hacia la obtención de un valor agregado o de conjunto de los diferentes bloques utilizados para evaluar la calidad peatonal, lo que permitiría comparar mejor entornos peatonales diferentes. Por otro lado, hay que llevar la investigación desde su actual dimensión exploratoria o descriptiva hacia una perspectiva más experimental, en la que técnicos competentes y profesionales del sector puedan aplicar el método CPEM y colaborar en su mejora. 


\section{Agradecimientos}

Este trabajo ha sido elaborado con la financiación de la Junta de Andalucía y los fondos FEDER para el proyecto de excelencia P09-RNM-5394: Guía metodológica para la integración metropolitana sostenible de los sistemas de metro ligero (INTEGRA-ME).

\section{Referencias bibliográficas}

Aguilera, F. (2008). Análisis espacial para la ordenación eco-paisajística de la aglomeración urbana de Granada. Granada: Universidad de Granada. Departamento de Expresión Gráfica, Arquitectónica y en la Ingeniería.

Alfonzo, M. A. (2005). "To Walk or Not to Walk?: The Hierarchy of Walking Needs». Environment and Behavior, 37, 808-836.

Alitoudert, F. y MAYer, H. (2006). "Numerical study on the effects of aspect ratio and orientation of an urban street canyon on outdoor thermal comfort in hot and dry climate». Building and Environment, 41, 94-108.

BAnister, D. (2008). "The sustainable mobility paradigm». Transport Policy, 73-80.

Bentley, I. (1999). Entornos vitales: Hacia un diseño urbano y arquitectónico más humano. Barcelona: Gustavo Gili.

Bertolini, L.; ClercQ, F. L. y Straatemeier, T. (2008). «Urban transportation planning in transition». Transport Policy, 15, 69-72.

Bertolini, L. y Dijst, M. (2003). «Mobility Environments and Network Cities». Journal of Urban Design, 8, 27-43.

Borst, H. C.; De Vries, S. I.; Graham, J. M. A.; Van Dongen, J. E. F.; BakKer, I. y Miedema, H. M. E. (2009). "Influence of environmental street characteristics on walking route choice of elderly people». Journal of Environmental Psychology, 29, 477-484.

CaO, X.; Mokhtarian, P. L. y Handy, S. L. (2009). «Examining the Impacts of Residential Self-Selection on Travel Behaviour: A Focus on Empirical Findings». Transport Reviews, 29, 359-395.

Carmona, M. (2003). Public places, urban spaces: The dimensions of urban design. Boston, MA: Architectural Press.

Cervero, R. (2002). "Built environments and mode choice: Toward a normative framework». Transportation Research Part D: Transport and Environment, 7, 265-284.

Cervero, R. y Kockelman, K. (1997). "Travel demand and the 3Ds: Density, diversity, and design». Transportation Research Part D: Transport and Environment, 2, 199-219.

Clifton, K. J.; Livi Smith, A. D. y Rodriguez, D. (2007). «The development and testing of an audit for the pedestrian environment». Landscape and Urban Planning, 80, 95-110.

Crane, R. (2000). «The Influence of Urban Form on Travel: An Interpretive Review». Journal of Planning Literature, 15, 3-23.

Delmelle, E. C. y CASAS, I. (2012). "Evaluating the spatial equity of bus rapid transit-based accessibility patterns in a developing country: The case of Cali, Colombia». Transport Policy, 20, 36-46.

EEA (2010). Good practice guide on noise exposure and potential health effects. Copenhage: European Environment Agency. 
Ewing, R. y Cervero, R. (2001). "Travel and the Built Environment: A Synthesis». Transportation Research Record: Journal of the Transportation Research Board, 1780, 87-114.

- (2010). "Travel and the Built Environment». Journal of the American Planning Association, 76, 265-294.

Ewing, R.; Clemente, O.; Handy, S.; Brownson, R. C. y Winston, E. (2005). "Measuring Urban Design Qualities Related to Walkability». Active Living Research Program. Robert Wood Johnson Foundation.

EWInG, R. y Handy, S. (2009). "Measuring the Unmeasurable: Urban Design Qualities Related to Walkability». Journal of Urban Design, 14, 65-84.

Foltête, J.-C. y Piombini, A. (2007). «Urban layout, landscape features and pedestrian usage». Landscape and Urban Planning, 81, 225-234.

Fruin, J. J. (1971). Pedestrian planning and design. Nueva York: Metropolitan Association of Urban Designers and Environmental Planners.

GEHL, J. (1971). Life between buildings: using public space. Copenhage: Danish Architectural Press.

Givoni, M. y Banister, D. (2010). Integrated Transport: From Policy to Practice. Londres: Routledge.

Handy, S. L. y Niemeier, D. A. (1997). "Measuring accessibility: An exploration of issues and alternatives». Environment and Planning A, 29, 1175-1194.

Hass-Klau, C. y Crampton, G. (2002). Future of urban transport: Learning from success and weakness: light rail. Wüppertal: Bergische Universität Gesamthochschule Wüppertal.

Herce, M. (2009). Sobre la movilidad en la ciudad: Propuestas para recuperar un derecho ciudadano. Barcelona: Reverté.

JACOBS, A. B. (1993). Great Streets. Cambridge, Mass.: Mit Press.

Landis, B.; Vattikuti, V.; OttenberG, R.; Mcleod, D. y Guttenplan, M. (2001). "Modeling the Roadside Walking Environment: Pedestrian Level of Service». Transportation Research Record: Journal of the Transportation Research Board, 1773, 82-88.

LeE, C. y Moudon, A. V. (2006). «The 3Ds+R: Quantifying land use and urban form correlates of walking». Transportation Research Part D: Transport and Environment, $11,204-215$.

LiTMAn, T. (2012). Well Measured-Developing Indicators for Comprehensive and Sustainable Transport Planning. Victoria: Victoria Transport Policy Institute, 35.

LOTFI, S. y KOOHSARI, M. (2009). "Analyzing Accessibility Dimension of Urban Quality of Life: Where Urban Designers Face Duality Between Subjective and Objective Reading of Place». Social Indicators Research, 94, 417-435.

LYNCH, K. (1981). La imagen de la ciudad. Barcelona: Gustavo Gili, 1960.

Manchón, L. F.; Santamera, J. A.; Diego, J. G. B. G. de; Mínguez, J. J. y OrmaZÁBAL, J. I. (1995). Recomendaciones para el proyecto y diseño del viario urbano. Madrid: Ministerio de Fomento.

NÆSS, P. (2009). «Residential Self-Selection and Appropriate Control Variables in Land Use: Travel Studies». Transport Reviews, 29, 293-324.

Nikolopoulou, M. y LyKoudis, S. (2006). «Thermal comfort in outdoor urban spaces: Analysis across different European countries». Building and Environment, 41, 1455-1470.

Olszewski, P. y Wibowo, S. (2005). «Using Equivalent Walking Distance to Assess Pedestrian Accessibility to Transit Stations in Singapore». Transportation Research Record: Journal of the Transportation Research Board, 1927, 38-45. 
Owen, N.; Humpel, N.; Leslie, E.; Bauman, A. y Sallis, J. F. (2004). «Understanding environmental influences on walking: Review and research agenda». American Journal of Preventive Medicine, 27, 67-76.

Peters, P. (1981). La ciudad peatonal. Barcelona: Gustavo Gili.

Pikora, T. J.; Giles-Corti, B.; Bull, F.; Jamrozik, K. y Donovan, R. (2003). «Developing a framework for assessment of the environmental determinants of walking and cycling». Social Science \& Medicine, 56, 1693-1703.

Pikora, T. J.; Giles-Corti, B.; Knuiman, M. W.; Bull, F. C.; Jamrozik, K. y Donovan, R. J. (2006). "Neighborhood Environmental Factors Correlated with Walking Near Home: Using SPACES. [Miscellaneous Article]». Medicine \& Science in Sports \& Exercise April, 38 (4), 708-714.

Portland, G. (1998). «Pedestrian design guide». Pedestrian Transportation Program. Portland: Office of Transportation Engineering and Development.

Pozueta, J. (2001). Instrucción para el Diseño de la Vía Pública en el municipio de Madrid. Madrid: Ayuntamiento de Madrid.

Pozueta, J.; Lamíquiz, F. J. y PORTO, M. (2009). La ciudad paseable: Recomendaciones para la consolidación de los peatones en el planeamiento, el diseño urbano y la arquitectura. Madrid.

Prinz, D. (1986). Planificación y configuración urbana. Barcelona: Gustavo Gili.

Raggam, R. B.; Cik, M.; HÖldrich, R. R.; Fallast, K.; Gallasch, E.; Fend, M.; LACKNeR, A. y MARTH, E. (2007). «Personal noise ranking of road traffic: Subjective estimation versus physiological parameters under laboratory conditions». International Journal of Hygiene and Environmental Health, 210, 97-105.

Rodríguez, D. A.; Brisson, E. M. y Estupiñán, N. (2009). "The relationship between segment-level built environment attributes and pedestrian activity around Bogota's BRT stations». Transportation Research Part D: Transport and Environment, 14, 470-478.

Salingaros, N. A.; Coward, L. A.; West, B. J. y Bilsen, A. (2005). Principles of urban structure. Amsterdam: Techne.

SANZ, A. (2008). Calmar el tráfico: Pasos para una nueva cultura de la movilidad urbana. Madrid: Ministerio de Fomento.

SARKAR, S. (2003). "Qualitative Evaluation of Comfort Needs in Urban Walkways in Major Activity Centers». Transportation Quarterly, 57, 39-59.

Sauter, D.; Hogertz, C.; Tight, M.; Thomas, R. y Zaidel, D. (2010). «Measuring Walking». En: Tight, M. y Walker, J. (eds.). Pedestrians' Quality Needs. Final Report of the COST project 358, Cheltenham: Walk21. <http://www.walkeurope. org/final_report/default.asp>

ShashUA-BAR, L. y HoffMAN, M. E. (2000). "Vegetation as a climatic component in the design of an urban street: An empirical model for predicting the cooling effect of urban green areas with trees». Energy and Buildings, 31, 221-235.

Silva, C. y Pinho, P. (2010). "The Structural Accessibility Layer (SAL): revealing how urban structure constrains travel choice». Environment and Planning A, 42, 2735-2752.

SORIA, J. (2011). «Modelo de umbrales para la evaluación ambiental de la movilidad urbana». Departamento de Urbanistica y Ordenación del Territorio. Granada: Universidad de Granada.

Soria, J. A.; Valenzuela, L. M. y Pinho, P. (2010). "A method to identify mobility environments in metropolitan transport corridors: a case study in Granada». Oporto, 4th CITTA Annual Conference on Planning Research. 
Stathopoulos, T.; Wu, H. y Zacharias, J. (2004). "Outdoor human comfort in an urban climate». Building and Environment, 39, 297-305.

Transport, G. B. D. F. (2007). Manual for Streets. Thomas Telford Pub.

Venturi, R.; Brown, D. S. y Izenour, S. (1977). Learning from Las Vegas: the forgotten symbolism of architectural form. Cambridge, MA: MIT Press.

Vieira, J.; Moura, F. y Manuel Viegas, J. (2007). «Transport policy and environmental impacts: The importance of multi-instrumentality in policy integration». Transport Policy, 14, 421-432.

Weinstein, A.; SchlossberG, M. y Irvin, K. (2008). «How Far, by Which Route and Why?: A Spatial Analysis of Pedestrian Preference». Journal of Urban Design, 13, 81-98.

ZACHARIAS, J. (2001). «Pedestrian Behavior and Perception in Urban Walking Environments». Journal of Planning Literature, 16, 3-18. 
